\title{
The Design and Implementation of Data System for Magnetic Alloys
}

\author{
Jing Wang ${ }^{1, a^{*}}$, Yu Bai ${ }^{2, b}$, Yan $\mathrm{Cao}^{3, \mathrm{c}}$ and Hu Qiao ${ }^{4, \mathrm{~d}}$
}

Department of Mechanical and Electronic Engineering, Xi'an Technological University, Xi'an, China 1033560085@qq.com, baiyv@xatu.edu.cn, jantonyz@163.com, qiaonwpu@hotmail.com

Keywords: Magnetic Alloy; Database system; JSP.

\begin{abstract}
Based on web, an integrated Magnetic alloys database system has been constructed, by adopting three-tier architecture such as B/S and using advanced technologies such as JSP and SQL. The system has several advantages such as friendly interface, simplicity, easy to use and understand, functional integrity that will help and reference to users to find the desirable data associated with products, to provide reference and data basis for product design.
\end{abstract}

\section{Introduction}

Magnetic alloy is a kind of material which is used to make functional devices with strong magnetism, magnetic heat and magnetic properties, it is widely used in the national defense and manufacturing, such as sensors, motors, MRI scanners, and electric vehicles [1]. In recent years, with the research of magnetic alloy, composition design and application, it is a tedious and inefficient work to find right information repeatedly in a large of literature data. Therefore, it has become a very realistic and urgent problem to use the database system to efficiently classify and store a large and number of complex data.

\section{Research Status of Material Database}

Research Status of Foreign Material Database. Material database is a set of application system, which is based on the analysis of materials and convenient application. The popularization of computer technology provides a wide range of development platform for the rapid development of database technology. There is a universal and convenient network of comprehensive and commercial database MatWeb in the US, which contains more than 580000 kinds of data, such as composite materials, metal materials, ceramics, and plastics [2]. It is convenient to search the data information in a variety of ways which are advanced search, properties, composition, name and manufacturer.

Research Status of Domestic Material Database. He Liping, the professor of Da Lian University of Technology, in the environment of Eclipse 3.4 and Java EE exploited a mold material database by using MySQLSever2008 and JDBC data interface [3]. Zhao Yakun, the professor of Huai Yin Institute of Technology and Huai Yin Normal University, on a basis of high speed machining technology, developed a database by using the Visual Basic language and SQL Server [4]. At present, it has not been yet built a corresponding database in the all kinds of magnetic alloys so each time using a material is required to search a data repeatedly in a document literature. Based on the present situation of the domestic database system, a database system of magnetic alloy is studied in this paper. The system can facilitate the selection of materials, scientific management of the category of magnetic alloy materials, brand, the main use of performance and other materials information to save time and cost for the development of magnetic alloys.

\section{The Design of Alloy Database System}

The Architecture of Database System. The function of material management system which is exploited by B/S multi layer distribution includes the customer layer, the Web layer, the business layer and the data access layer. In an Internet environment, a personal computer interactive product design platform which based on the development language of Java has been completed by using a 
popular JSP dynamic web [5]. Fig. 1 for the three-tier architecture B/S in which we can connect a database network with the JDBC connection method. JDBC provides a seamless operation of a variety of data interface for the Java program to automatically transfer SQL to the corresponding database management system.

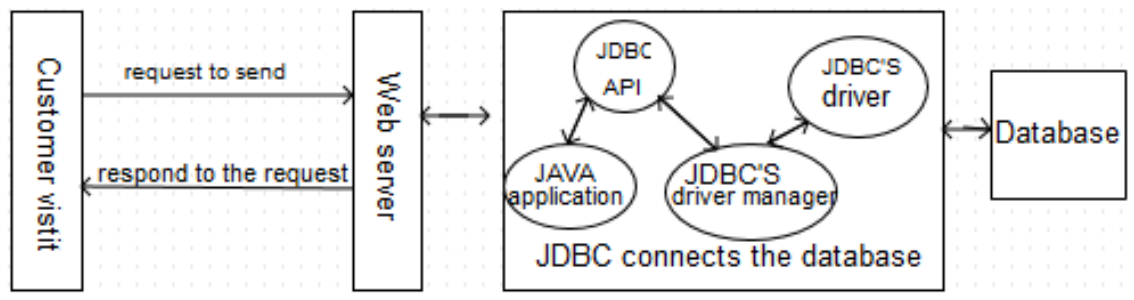

Figure 1. The three-tier architecture B/S

\section{The Development Environment of Database.}

Operating system: Windows 7.

Development platform: Java JDK1.6.

Development tool: JSP.

Database: SQL Server2008.

Hardware environment: CPU 1.8 G, Memory 2G, Hard disk 160G.

\section{Database Structure.}

Design of conceptual structure. The data which has a very high reliability in this system is from the China Aviation Handbook fifth volumes. According to the difference of magnetic induction, the information can be divided into 14 alloys which are electromagnetic pure iron, electrical sheet and soft magnetic alloy of iron and nickel system and 191 brands. Each alloy has a corresponding information which are material trademark, technical criterions of materials, physical properties (density, thermal properties, electrical properties and magnetic properties), chemical properties, mechanical properties (bending properties, tensile properties), organization structure, process performance and requirements, the use of suggestions. Rectangular, oval and prism are on behalf of entity, property and relation respectively in the conceptual model design phase. As far as the database is concerned, the entity refers to the different alloy grades and the properties refer to all kinds of alloy performance of database. According to the demand analysis, you can get the entity attributed graph and the E-R diagram of system database as shown in Fig 2.

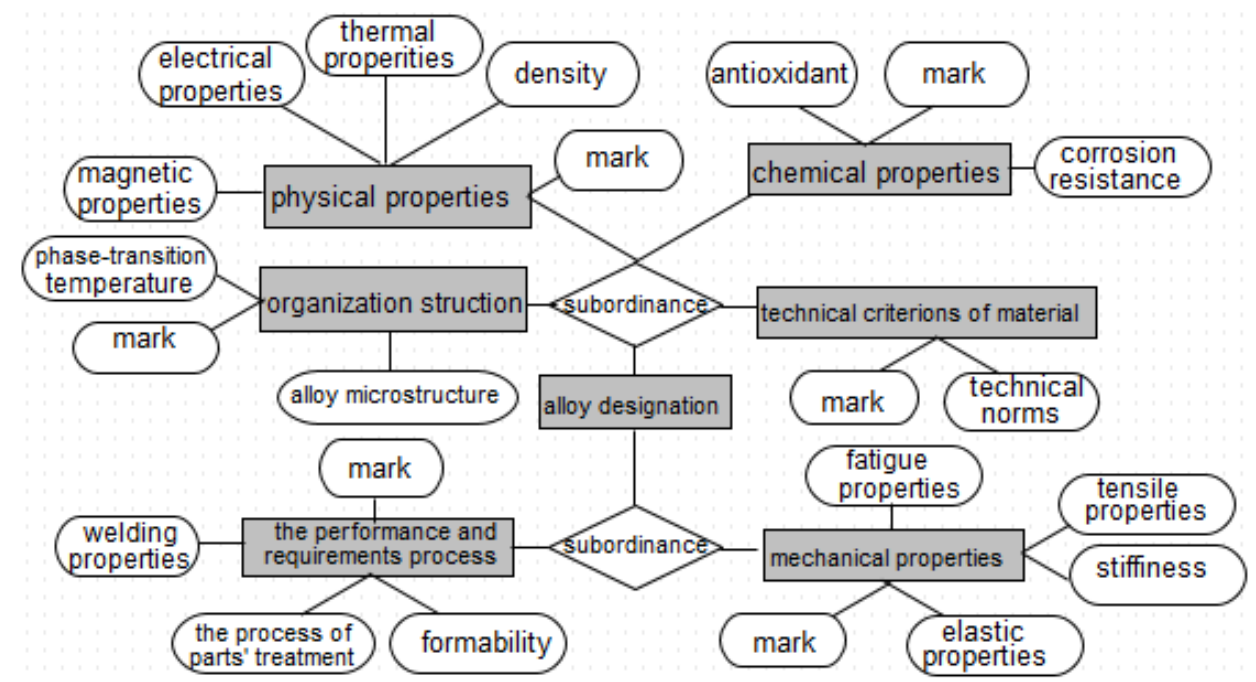

Figure 2. Entity relation diagram of magnetic alloy database

Design of Logical structure. The system which uses a relational database management system Sever SQL 2008 is generally regard a primary key as an index, so you can set a field as an index. 
The type and length of a field generally depends on the type and content of the data to be stored. According to the general information of the magnetic alloy, the E-R diagram of the conceptual design stage is changed into the relational model in the database logic design stage. The relationship model is composed of a number of data tables which are set up according to the standard of a three-normal form and a relation corresponds to a table in the database ${ }^{[6]}$. E-R model is transformed into each part of the entity and each date shows in Table 1

Table 1 Physical properties

\begin{tabular}{cccccc}
\hline Field name & Type & $\begin{array}{c}\text { Lengt } \\
\mathrm{h}\end{array}$ & Not null & $\begin{array}{c}\text { Windows } \\
\text { default }\end{array}$ & Explanation \\
\hline Mark & Nvarchar & 20 & Not null & & Main index field \\
Electrical properties & Varchar & 20 & Null & & \\
Magnetic properties & Varchar & 20 & Not null & & \\
Thermal properties & Varchar & 20 & Not null & & \\
Density & Varchar & 20 & Not null & & \\
\hline
\end{tabular}

\section{The Function Module of Database System}

Database Structure Designing. A wide range of magnetic alloy materials are studied carefully and classified scientifically, and then you can use the structured system analysis and design ideas in this system. At the top of the system, it is parted into a reasonable level to divide the complex system into relatively independent, single function module. The overall functional structure of the system is shown in Fig. 3, including material selection, database maintenance, user management, supplier management and system help. For security reasons the system is divided into management in the design process, which means different users are set different permissions.

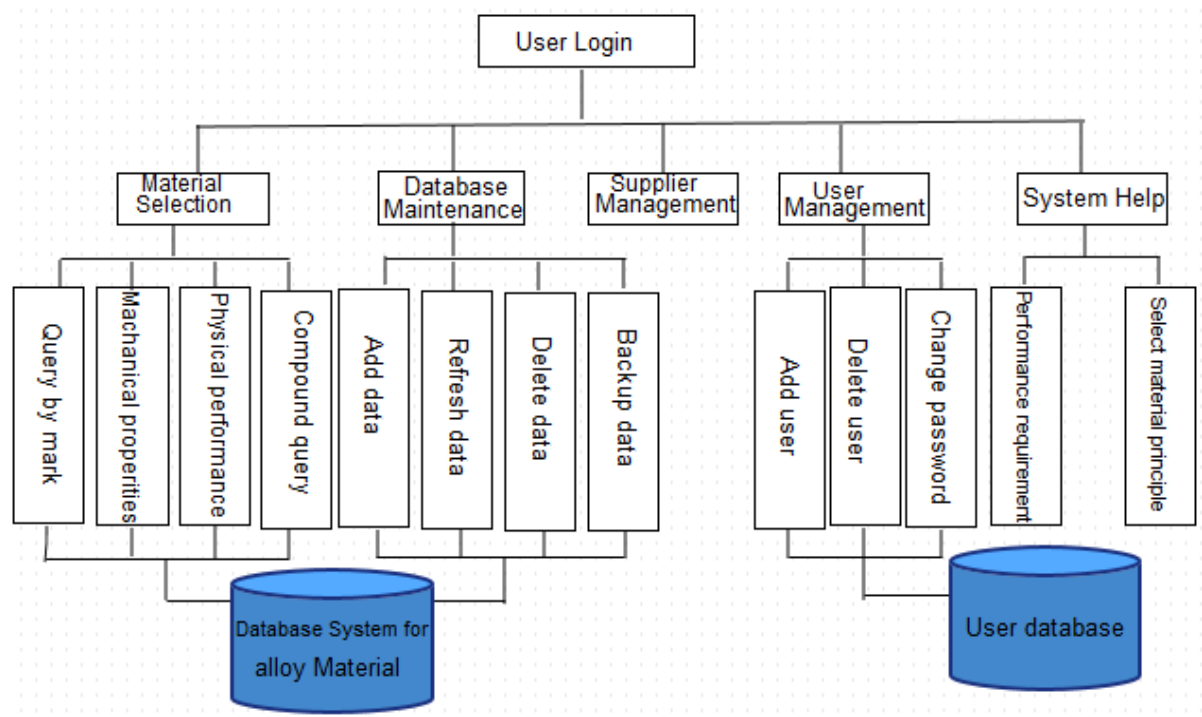

Figure 3. System structure and function

Key Technology of System Development. This system which is achieved by a B/S mode uses My Eclipse as a development tool and Server2005 SQL as the background database.

Hardware environment: it is recommended to use $1 \mathrm{GHzPentium}$ processor, but using Minimum Pentium 600MHz processor and operating system uses Windows SP3/XP SP2/2003 SP1 Microsoft and above. The minimum memory requirements $512 \mathrm{MB}$, the minimum requirement for the display is $1024 * 768$ and the system drives to have enough free space to ensure the stability of the system. 
Software environment: please install JDK, My Eclipse development tools, tomcat server and Server SQL 2005 database.

System Login Interface. Because this system is aimed at the magnetic alloy products, some of the pictures and properties of common magnetic alloy are showed on the home page. There are main features of the system, login, registration and other information in the home page. As shown in Fig 4, users can see the system's login, registration, material selection, database maintenance and related alloy database links in this system. Only registered users can further carry out the information of the query. After registering the system, the user is in accordance with their respective rights to achieve the operation of the system.

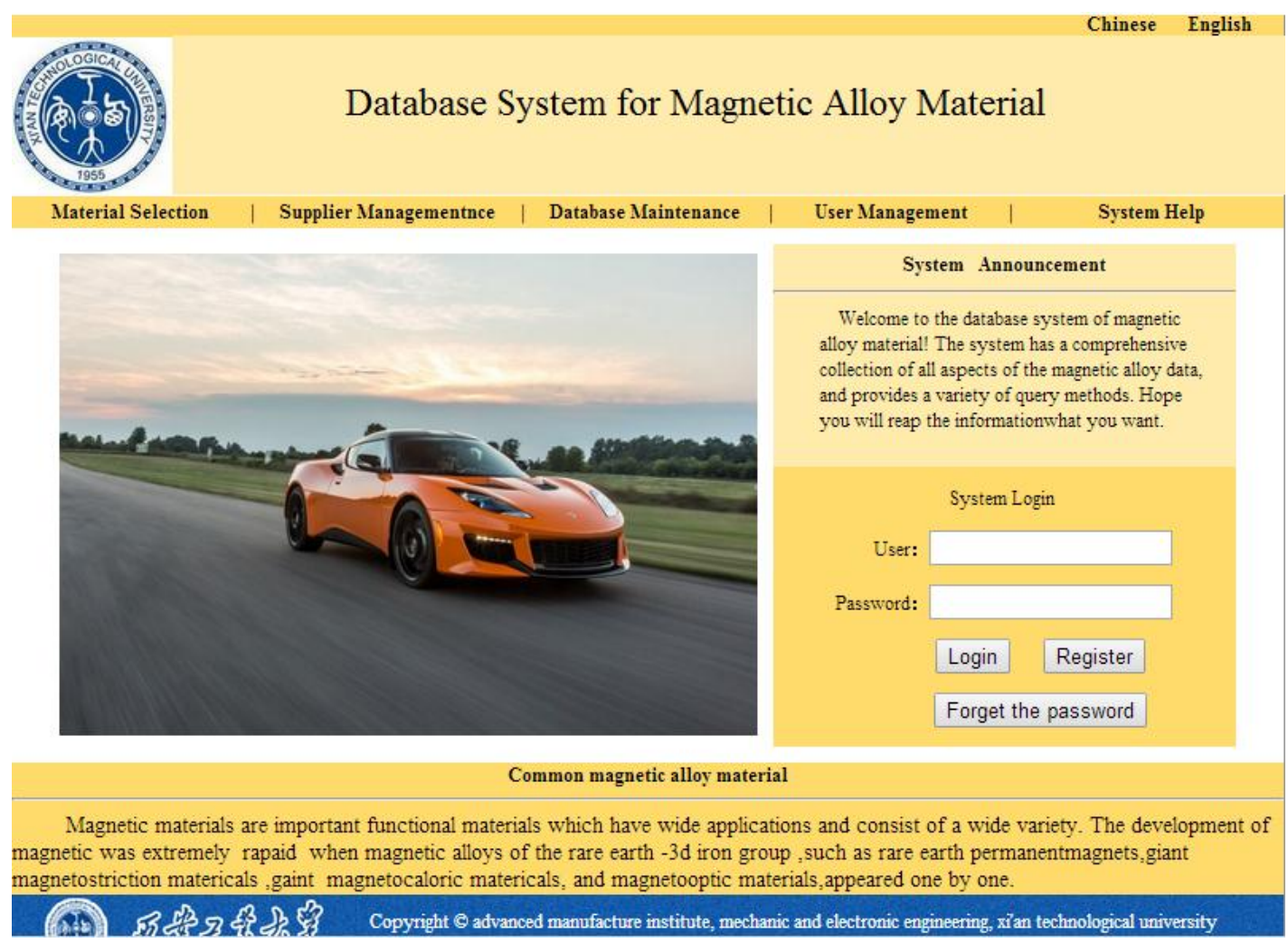

Figure 4. The main interface of system

System Query Page. Click on the Material inquiry into a query page as shown in Fig. 5 and according to the main system of magnetic properties you can find kinds of queries: query by grade, query by physical performance, query by mechanical properties and Compound query in system. Clicking on the "query by grade" the system appears to enter the alloy grade dialog box and system will output all the data that meets the conditions in the database when users input the mark and click the "start". For example, the user inquires the grade of DT3 magnetic alloy and the system will return the results of the query. 


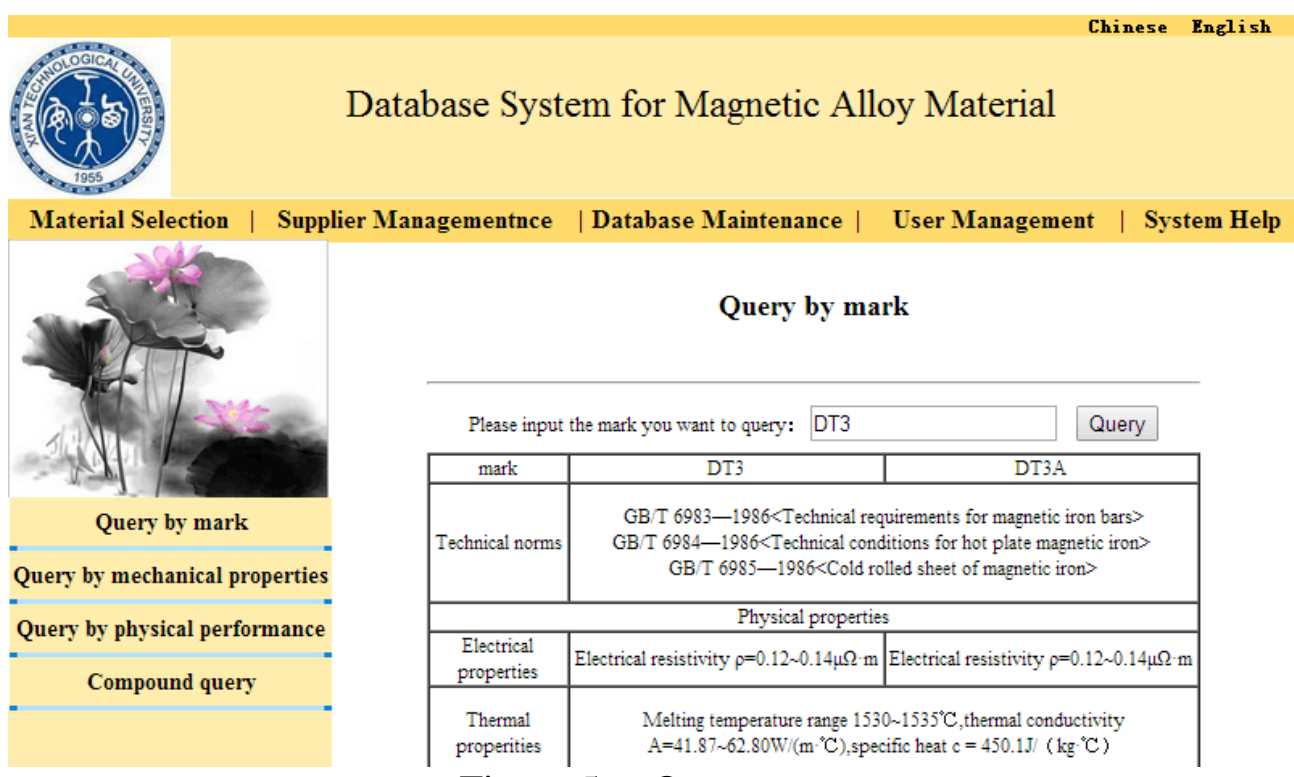

Figure 5. Query page

System Maintenance Function Module. System maintenance module which includes the database of material information to add, modify, delete and other operations is developed for the system administrator to maintain the database effectively and timely. The function of database system can be enriched and improved in the use of the process and through interactive operation users can manage and maintain the Magnetic alloy material database, so that the data can be expanded. In order to ensure the security and reliability of the data information, you can add, modify and delete the data in the database only if you are an administrator. Otherwise, the user has no right to use this function to manage the data information. Fig 6 shows the DT3A magnetic alloy data information to modify the interface.

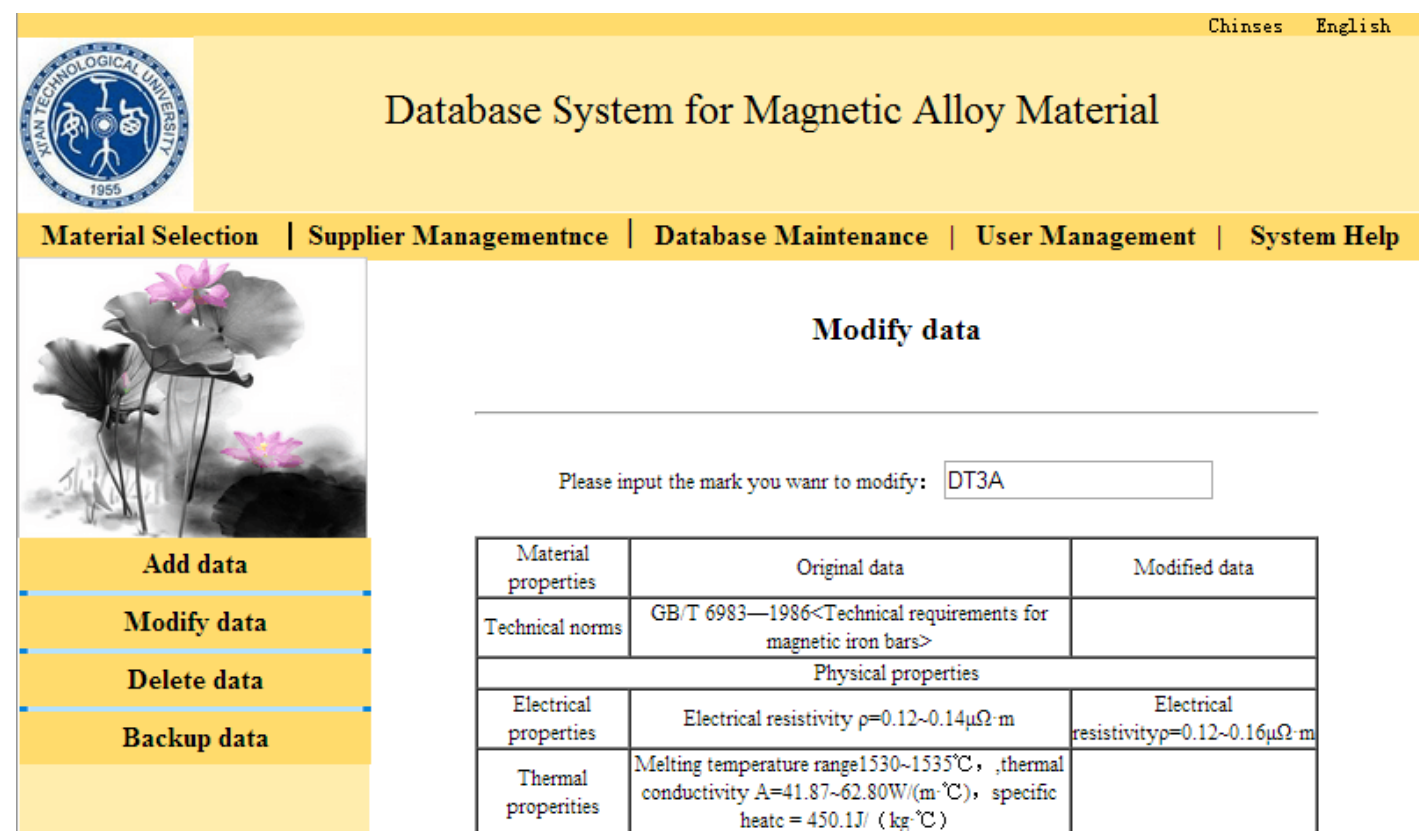

Figure 6. Interface of data information modification

\section{Summary}

Using JSP as the development tool, Java as the system function module and Html technology to set up the system page, combined with the database to develop a more complete set of magnetic alloy 
database system. Through the Internet you can complete the database system access and query and other operations and broaden the user access to magnetic alloys and related information channels.

The information is complete and the data source credibility is high because all of date is from the China Aviation Handbook fifth volumes and have a professional analysis and sorting and repeated check to ensure the quality of the data and standardization before inputting the database.

System interface is friendly to easily operate. By a "thin client" technology, users only need a browser to be able to quickly access through the Internet and have no need to install other applications.

The system function is complete and the maintenance is convenient. The system not only provides the function of access and query, but also designs the supplier and transportation module. This system not only achieves the interaction of itself and the outside world, but also provides appropriate suppliers improving the efficiency of the actual production when you consult data

\section{Acknowledgments}

The paper is supported by Key Problem Tackling Project of Shaanxi Scientific and Technological Office (2016GY-024) and non-traditional machining Key Laboratory Project of Shaanxi Province (15JS041).

\section{References}

[1] Chengbao Jiang, Jingmin Wang, Huibin Xu. Progress of Magnetic shape memory Alloy [J]. Progress of materials in China, 2011, 09:42-50+56.

[2] Sun Yu, Weidong Zeng, Yuanfei Han, Tengfei Wang, ZHAN Yongqing. Design and development of database system for titanium alloys [J]. The Chinese Journal of Nonferrous Metals, 2010, S1:447-451.

[3] Du Yuan. The Design and Implementation of Data Management System for Alumium Alloys [D]. GuangXi University, 2014.

[4] Yakun Zhao, Hongbo Tan, Ling Guo, Xiangdong Chu, Chengcheng Lu, Tongrui Wang. Design and function realization of high speed machining database [J]. Science and Technology Innovation Herald, 2008, 13:27-28.

[5] Wenxue Fan. Research on Technology of Network Manufacture in Workshop Based on the CAXA Engineer [D]. Inner Mongolia University of Technology, 2007.

[6] Lei Zhang. Research and development of wrought magnesium alloy data development platform [D]. Chongqing University, 2015.

[7] Wei Li, Jintao Gao, Teng Gao. Relational Database Keyword Search on E-R Diagram[J]. Application of computer system, 2012, 09:156-161+140.

[8] Qinlong Wang. Art colleges and Universities Based on The JSP Library Management System Design and Implementation [D]. Xidian University, 2013.

[9] Zhiqiang Yuan, Ai-Tao Tang, Hai-Ding Liu, Chuan-Pu Liu, Biao Xu. Design and Development on magnesium Alloys Database Based on Web [J]. Journal of Chongqing University (Natural Science Edition), 2007, 02:94-97.

[10]Xingxing Xue, Ri Li, Xueliang Liu, Ruishi Wu. Design and Development of Cast Aluminum Alloy Database [J]. Foundry, 2009, 12:1238-1240. 